\title{
VaR Trend Analysis from Discretized and Continuous Approaches
}

\author{
M. Maïza, A. Clavel, N. Hatem, and C. Recommandé
}

\begin{abstract}
Present paper objective is to analyze how trends can be detected and interpreted using a $\operatorname{GARCH}(1,1)$ model. Through VAR calculation along 2 distinct ways, the whole concept is to find out if trends can be identified in the return series of an index. Then this information can be used with an estimated probability to complement the VAR in order to get better anticipation of possible losses in a stressed environment. In addition, back testing on CVaR reliability compared to the VaR will be run as well.
\end{abstract}

Index Terms-CVaR, estimated shortfall, GARCH, model comparison, portfolio management, trend analysis, VAR.

\section{INTRODUCTION}

The GARCH model - for Generalized Autoregressive Conditional Heteroskedasticity - has been introduced and improved by Engle and Bollerslev [1]. Initially mono variable, the model was acceptable enough to compute data from a single asset or index alone. However when applied to full index or portfolio, by far the most common case for this kind of model, the results given by GARCH model can be questionable.

As it does not provide each individual return of the assets composing the index, the model cannot integrate a correlation between all their variations. Furthermore, the size of the index or portfolio can matter as well, because of possible correlation issue, and systemic risk gets higher.

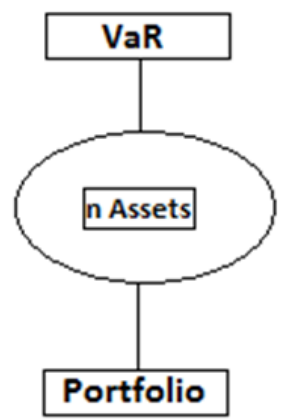

Fig. 1. VaR Calculation with Univariate GARCH model.

Either way GARCH model will provide a computed volatility which can be used to compute the Value at Risk (VaR) [2] Fig. 1 highlights that such a calculation with GARCH $(1,1)$ model can be only run on the return of

Manuscript received March 26, 2015; revised July 5, 2015. This work was supported in part by the ECE Paris under its PFE program for undergraduate students.

M. Maïza, A. Clavel, N. Hatem, and C. Recommandé are with the ECE Paris, Paris, 75015 France (e-mail: maiza@ece.fr; clavel@ece.fr; hatem@ece.fr; recommand@ece.fr). portfolio or index. For better index modeling [3], a calculation run with DCC-GARCH model can integrate return variations of different assets in the portfolio through their correlation matrix [4].

But instead of trying to use DCC-GARCH model approach [5], the study will focus on selecting indexes of different size, so that the model used on a small index could react as if it were in its multivariate form. Indeed in a small index, assets may be more correlated, so their variations will much more impact overall index return, which would not be as much noticeable with an index composed of hundreds of assets.

\section{PROBLEM DESCRIPTION}

Since 2008, world economic crisis has raised the need of improved estimation of upcoming portfolio returns or index. Indeed, numerous models available to run simulations on a portfolio all have their plus and cons, and GARCH model is the most common one [6].

Though even in its DCC form, GARCH model provides better portfolio modelling [7]; better sensitivity to systemic risk may not be accurate enough in a stressful environment. During a crisis period where fluctuations are very large, volatility calculation might not be so accurate, and systemic risk, as it is a rather new aspect taken in consideration, might remain uncertain.

Thus major criticism regarding expected loss, calculated with $\mathrm{VaR}$, is the specific issue of systemic risk estimation. Indeed while the economy runs smoothly, VaR calculation may provide accurate estimation of portfolio possible loss. But in crisis time, limits of any model are under question.

This is why, with so little back test, developing the approach of conditional VaR [8], aka CVaR calculation, is absolutely critical. Indeed with proper back testing on data of last decade, simulations can provide valuable results regarding $\mathrm{CVaR}$ accuracy to estimate upcoming shortfall of a portfolio or index during crisis time [9].

Furthermore, this will allow develop a new approach on GARCH model, which is to identify trends in CVaR, VaR and any model output. So in addition to taking into consideration the risk showed by $\mathrm{VaR}$ and $\mathrm{CVaR}$ aside, analysis of the trends could bring (if a variable probability) an additional measure of risk.

This new approach is known to have a real potential, because econometric models only have what can be called "horizontal" risk measure as they only use data at time t. Actually some can be used with a couple of periods, but it is a real difficulty to set the correct parameters. This is why trends, which will be identified on 3 periods, can bring a useful vertical approach to the model itself. 


\section{BASE MODEL}

\section{A. Review Stage}

To make accurate predictions related to possible loss of portfolio or index, and to test GARCH model efficiency, VaR and $\mathrm{CVaR}$ parameters computed with GARCH model will be first compared with the ones obtained by using raw data and basic formulae. As indicated earlier, the idea is to identify in a second time trends with a high probability of recurrent patterns. This can be adapted either to index returns, or to VaR and CVaR when the index is overwhelmed.

The 3 main equations of GARCH $(1,1)$ model [10] which is the backbone of present analysis are given by

$$
\begin{gathered}
r_{t}=\mu_{t}+a_{t} \\
a_{t}=h_{t}^{1 / 2} z_{t} \\
h_{t}=\alpha 0+\alpha 1 a_{t-1}^{2}+\beta 1 h_{t-1}
\end{gathered}
$$

where $\alpha, \alpha 1$ and $\beta$ are model parameters. In (1) $r_{t}$ refers to the $\log$ return, $\mu_{t}$ to the expected value of $r_{t}$ and $a_{t}$ to the mean corrected return. In (2) $h_{t}$ is the square of volatility and $z_{t}$ are independent and identically distributed random variables.

Attention is focused on $\operatorname{GARCH}(1,1)$ model as it is most reliable and easier to compute. Indeed as the final goal is to identify trends on a 3 periods interval, $\operatorname{GARCH}(3,3)$ model would also provide outputs on the interval. However this later model is excessively difficult to work with as parameters $\alpha$ and $\beta$ will be particularly hard to determine using common solvers.

CVaR calculation more specifically is based on the VaR here evaluated either directly from data or used with GARCH model in its parametric form. The parametric approach stands for a calculation with data variance and covariance. CVaR can be further developed, and its calculation implies a couple of steps. Actually first step is to get the measures of profit and loss of 2 portfolios or indexes. Then to obtain the joint law of these measures $-X($.$) and Y($.$) here with x_{n}$ and $y_{n}$ the $P n L$ measures at day $n$ :

$$
\begin{aligned}
& \left\{p^{(X Y)}(i, j)=\mathbb{P}\left[X(.)=x_{i}, Y(.)=y_{j}\right] \mid i \in\{1, \ldots, I\}, j \in\right. \\
& \{1, \ldots, J\}\}
\end{aligned}
$$

Next step consists in obtaining the performance of only one portfolio:

$$
p_{i}^{(X)}=P\left[X(.)=x_{i}\right]=\sum_{j=1}^{J} p^{(X Y)}(i, j)
$$

Assuming that $x_{m}(x)$ and $y_{m}(y)$ are the respective values of greatest negative performance of each portfolio, the random variable loss_X(.) takes its values in $\{O$, $\left.\lambda_{1}^{(x)}, \ldots, \lambda_{i}^{(x)}, \ldots, \lambda_{m^{(x)}}^{(x)}\right\}$ and:

$$
p^{\left(\operatorname{loss}_{-} X\right)}(i)=p^{(X)}\left(m^{(x)}-(i-1)\right)
$$

Assuming that $x_{m}(x)$ and $y_{m}(y)$ are the respective values of greater negative performance of each portfolio, loss_X(.) takes its values in $\left\{O, \lambda_{1}^{(x)}, \ldots, \lambda_{i}^{(x)}, \ldots, \lambda_{m^{(x)}}^{(x)}\right\}$

$$
\begin{gathered}
p^{\left(\operatorname{loss}_{-} X(.)\right)}(0) \equiv P\left[\operatorname{los}_{X(.)}=0\right]=\sum_{i=1}^{m^{(x)}} p^{(X)}(i) \\
p^{\left(\operatorname{loss} \__{-}(.)\right)}(i) \equiv P\left[\operatorname{loss}_{X(.)}=\lambda_{i}^{(x)}\right]=P[X(.)= \\
x m x-i-1=p X(m x-i-1)
\end{gathered}
$$

Fixing the system under normal market conditions, the event $C_{\text {norm }}$ can be defined as:

$$
C_{\text {norm }}(Y)=\left\{\mu_{(Y)}-v_{\sigma_{(Y)}} \leq \mu_{(Y)}+v_{\sigma_{(Y)}}\right\}
$$

From the joint distribution, the limits of discretized intervals are

$$
\left\{\begin{array}{l}
\theta_{(Y)}^{-}=\mu_{(Y)}-v_{\sigma_{(Y)}} \\
\theta_{(Y)}^{+}=\mu_{(Y)}+v_{\sigma_{(Y)}}
\end{array}\right.
$$

Thus $C_{\text {norm }}$ event probability can be reduced to the following simplified expressions:

$$
\begin{aligned}
& P\left[C_{\text {norm }}(Y)\right]=\sum_{j=m_{-}^{(Y)}+1}^{m_{+}^{(Y)}} p^{(Y)}(j) \\
& P\left[X(.)=x_{i}, C_{\text {norm }}(Y)\right]=\sum_{j=m_{-}^{(Y)}+1}^{m_{+}^{(Y)}} P[X(.)= \\
& x i, Y .=y j]=j=m-Y+1 m+Y p X Y i_{j} j
\end{aligned}
$$

from which expected value $\mathrm{X}()$ can be calculated conditioned by the event $C_{\text {norm }}(Y)$ :

$$
\begin{gathered}
\mathrm{E}\left[X(.) \mid C_{\text {norm }}(Y)\right]=\sum_{i=1}^{I} x_{i} P\left[X(.)=x_{i} \mid C_{\text {norm }}(Y)\right]= \\
\left(P\left[C_{\text {norm }}(Y)\right]\right)^{-1} \sum_{i=1}^{I} x_{i} P\left[X(.)=x_{i}, C_{\text {norm }}(Y)\right]
\end{gathered}
$$

On the other hand when considering a stressed market, the calculation is quite the same except for interval limits: lower limit is now defined by $\min (Y()$.$) and upper limit by \operatorname{VaR}(\mathrm{N}$ days, $99 \%$ ).

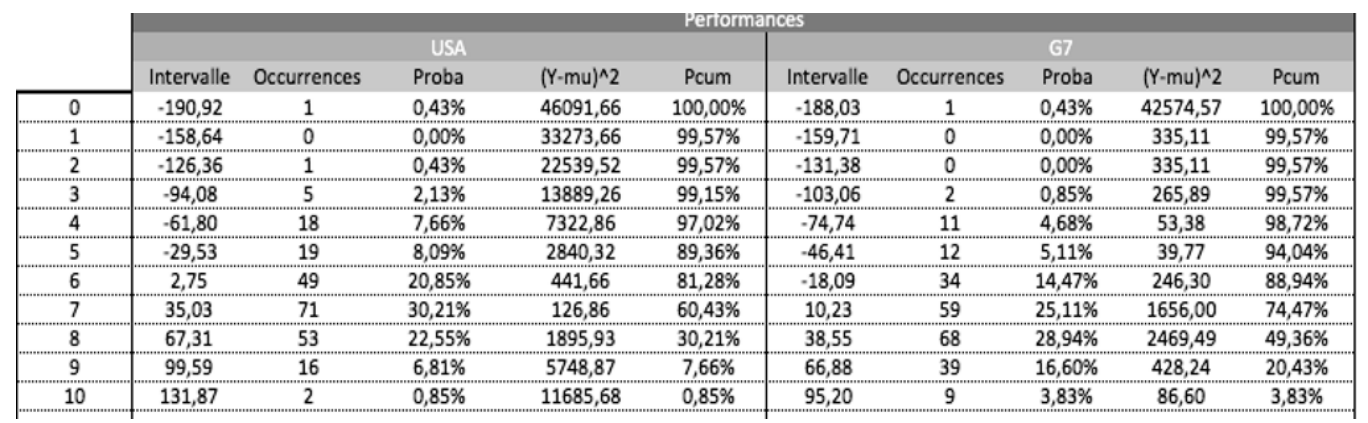

Fig. 2. Filled intervals using discretized approach. 

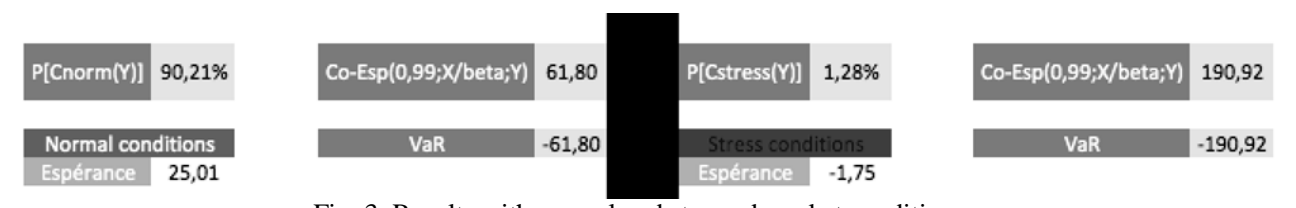

Fig. 3. Results with normal and stressed market conditions.

\begin{tabular}{|c|c|c|c|c|c|c|c|c|c|}
\hline \multicolumn{10}{|c|}{ Résumé PTF } \\
\hline \multirow{2}{*}{ PTF } & \multicolumn{3}{|c|}{ Infos PTF } & \multicolumn{3}{|c|}{ Normal conditions } & \multicolumn{3}{|c|}{ Stress conditions } \\
\hline & Espérance & Variance & Var & Espérance & Co-Expected shortfall & Co-VaR & Espérance & Co-Expected shortfall & Co-Var \\
\hline USA & 7,62 & 2345,65 & $-116,47$ & 3,08 & 42,02 & $-42,02$ & $-1,93$ & 190,92 & $-190,92$ \\
\hline UK & 6,22 & 3920,54 & $-169,78$ & 3,83 & 76,06 & $-76,06$ & $-2,29$ & 218,37 & $-218,37$ \\
\hline France & 0,52 & 29,98 & $-14,68$ & 0,37 & 10,33 & $-10,33$ & $-0,17$ & 14,68 & $-14,68$ \\
\hline Canada & 8,17 & 3019,82 & $-171,12$ & 4,35 & 43,47 & $-43,47$ & $-2,76$ & 254,89 & $-254,89$ \\
\hline Italy & 0,23 & 21,84 & $-12,16$ & 0,11 & 8,42 & $-8,42$ & $-0,15$ & 15,22 & $-15,22$ \\
\hline Allemagne & 0,55 & 33,13 & $-18,95$ & 0,41 & 6,09 & $-6,09$ & $-0,19$ & 19,80 & $-19,80$ \\
\hline
\end{tabular}

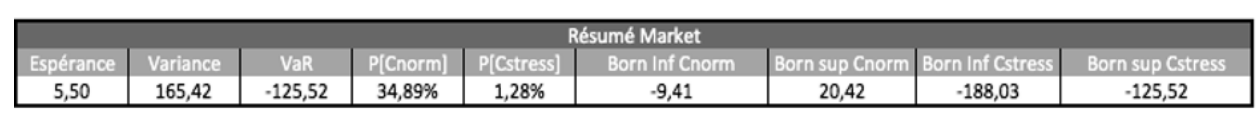

Fig. 4. Overall VaR calculation with discretized approach.

\begin{tabular}{|c|c|c|c|c|c|c|}
\hline \multirow[t]{6}{*}{$\underline{\text { Moments }}$} & Mean & $0,005 \%$ & & Paramètres & Alpha & $6,33 \mathrm{E}-06$ \\
\hline & Variance & 0,000181757 & & & Alpha1 & 0,200000443 \\
\hline & Skewness & 0,018391196 & & & Betal & 0,7999995557 \\
\hline & Kurtosis & 3,61 & & & Conditions & 1 \\
\hline & Standard Deviation Mean & $1,36 \%$ & & & & Alpha Alpha1 \& Beta $1>0$ \\
\hline & Smoothing Parameter & 0,94 & & & & \\
\hline \multicolumn{7}{|c|}{ Calcul de Volatilité du CAC 40 en supposant que la V } \\
\hline Close & Log Close & Return & Return $^{2}$ & Conditional Variance & Likelihood & Conditional Standard Deviation \\
\hline 4013,97 & 8,30 & & & & & \\
\hline 4012,91 & 8,30 & $-0,03 \%$ & $0,000 \%$ & 0,000181757 & 8,612469175 & 0,013481727 \\
\hline 4017,67 & 8,30 & $0,12 \%$ & $0,000 \%$ & $=\$ H S 2+S H S 3^{*} E 12+S H S 4$ & 8,784031948 & 0,012318677 \\
\hline 4024,80 & 8,30 & $0,18 \%$ & $0,000 \%$ & 0,000128011 & 8,938851945 & 0,011314204 \\
\hline 4045,14 & 8,31 & $0,50 \%$ & $0,003 \%$ & 0,000109368 & 8,888468622 & 0,010457917 \\
\hline 4043,09 & 8.30 & $-0,05 \%$ & $0,000 \%$ & $9,8907 \mathrm{E}-05$ & 9.218756584 & 0,009945198 \\
\hline
\end{tabular}

Calcul de VaR Paramétrique Normal Distribution

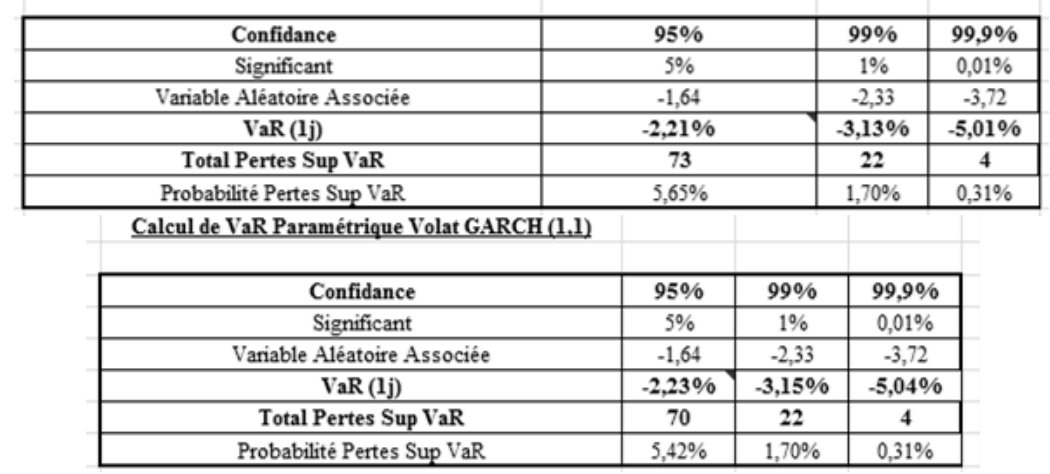

Fig. 6. VaR Calculation with $\operatorname{GARCH}(1,1)$

\section{Simulations}

The simulation process is based on a non-constant daily volatility computed with GARCH $(1,1)$ model [11]. Daily $\mathrm{VaR}$ can be computed with parametric formula using historical data over 5 years. Using discretized approach, VaR calculations are also run on a daily basis using the same data. They go through different steps, first one compute all asset returns. Next step is to identify all the intervals for the simulations and fill them with data; this is what Fig. 2 depicts with the intervals from 0 to 10 that are used for the computation, going along with the formulas.

Then $C_{\text {norm }}$ and $C_{\text {stress }}$ limits are determined from (10). After computing occurrence matrix, $C_{\text {norm }}$ and $C_{\text {stress }}$ are calculated.

Running the process with 6 out of the 7 indexes composing the MSCI World index, the following results are obtained, see Fig. 4, from market close data over 10 years period.

Before proceeding to trend analysis, it is interesting to note that, from these results, $\mathrm{VaR}$ is always located between Normal and Stressed CVaR. Now the focus will be set on simulation using GARCH model.

As noticeable on Fig. 3, GARCH model is used with Excel, so model parameters are obtained with its solver. Fig. 5 depicts another set of GARCH formulas and its parameters. On Fig. 6 there are the calculus run with the model showed in Fig. 5.

\section{TREND ANALYSIS}

First trend analysis relies heavily on accuracy of previous calculations and efficiency of actually run back testing. Thus 
in order to start identifying trends and to look for patterns in obtained outputs, the first step is to create normal conditions for the test index in the same way as for the market. They can be categorized in four classes:

Once all different performances have been ranged using these 4 classes as showed in Fig. 7, trend detection starts by analyzing the data on performances at time $t+1$ and $t+2$ for each time $t$.

From Fig 8 and 9 one trend has been highlighted. When benefits are higher than market upper limit, index performance is situated in the normal market for the following 2 days. This means that if Class 4 is encountered at a time $\mathrm{t}$, then Class 3 scenario will occur at times $t+1$ and $t+2$. This trend is identified here with a $100 \%$ precision.

In addition, with $71 \%$ precision, if performance is located in normal market at time $t$, it will remain in at times $t+1$ and $t+2$. In other words, there is $71 \%$ possibility to stay in Class 3 scenario 3 days in a row.

\begin{tabular}{|l|l|}
\hline Class 1 & Loss superior to the VaR \\
\hline Class 2 & $\begin{array}{l}\text { Loss located between the VaR and the lower limit of the } \\
\text { normal market }\end{array}$ \\
\hline Class 3 & Performance located in the normal market \\
\hline Class 4 & Benefits higher than the upper limit of the normal market \\
\hline & Fig. 7. Custom classes for trend analysis.
\end{tabular}

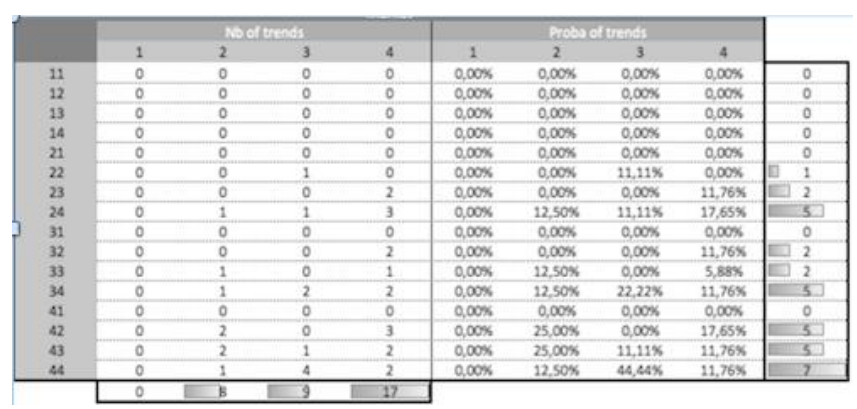

Fig. 8. Results of first trend analysis.

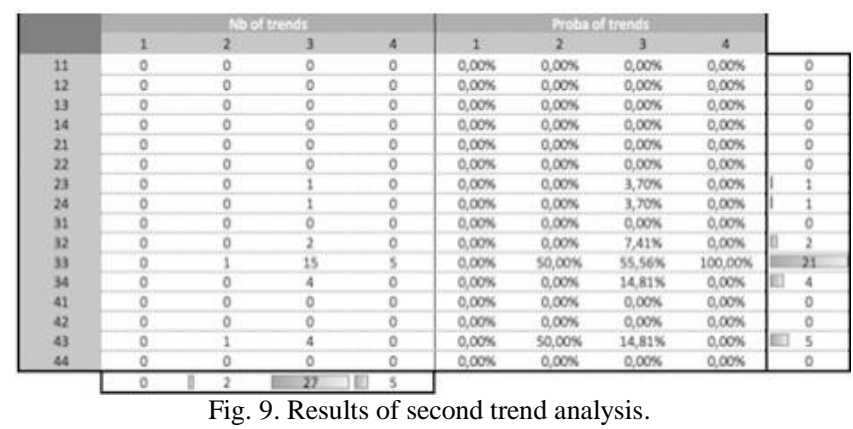

\section{CONCLUSION}

To strengthen prediction capability of losses for portfolios and indexes, an approach based on trend detection resulting from improved GARCH model has been proposed. The method is using finer evaluation of $\mathrm{VaR}$ and $\mathrm{CVaR}$ in different market conditions. It has been possible here to identify one trend with very high probability, though more data and more calculations are needed to ascertain exact result reliability. This would allow extend the analysis and not just work on a reduced sample of the market. Actually this is the main important issue, because identification, when adding this new set of predictions tools, is the first step of a process which represents a useful complement to actual econometric models relying only on reaction,.

\section{ACKNOWLEDGMENT}

The authors would like to thank ECE Paris Graduate School of Engineering for having provided the environment and support needed for present study. They are also indebted to $\operatorname{Pr}$ Y. Rakotondratsimba for his guidance during the research and Pr M. Cotsaftis for help in preparation of the manuscript.

\section{REFERENCES}

[1] B. Pesaran and M. H. Pesaran, "Modelling volatilities and conditional correlations in futures markets with a multivariate $t$ distribution," CESIFO Working Paper no.2056, categorie 10, 2007.

[2] C. Dadi and D. Ciolac, "Prédiction de la VaR basée sur GARCH et méthode de correction," Paris VII University, 2013.

[3] D. Yang and A. Schwert, Crisis Period Forecast Evaluation of the DCC-GARCH Model, North Carolina: Duke University Durham, 2010.

[4] R. Engle and K. Bryan, "Dynamic Equicorrelation," Journal of Business \& Economic Statistics, vol. 30, no. 2, pp. 212-228, 2012.

[5] E. Orskaug, Multivariate DCC-GARCH Model - With Various Error Distributions, Norvegia: Norsk Regnesentral, June 2009.

[6] V. Boisbourdain, Modèles GARCH et Risques de Marché, OTC Conseil France, 2008.

[7] E. Chiasson, "Valeur exposée au risque: Estimations par des modèles de corrélations conditionnelles dynamiques," Ph.D. dissertation, Université du Québec à Montréal, July 2012.

[8] B. Pesaran and M. H. Pesaran, "Conditional volatility and correlations of weekly returns and the VaR analysis of 2008 stock market crash," Economic Modelling, vol. 27, 2010.

[9] O. Roustant, Modèle GARCH: Application à la Prévision de la Volatilité, France: Ecole des Mines de Saint-Etienne, December 2007.

[10] C. Francq and J.-M. Zakoïan, GARCH Models: Structure, Statistical Inference and Financial Applications, UK: Wiley, 2010.

[11] T. Nakatani, "Ccgarch: An R package for modelling multivariate GARCH models with conditional correlations," Dept. of Agricultural Economics, Hokkaido University, Japan, Dept of Economic Statistics, Stockholm School of Economics, Sweden, August 12, 2008.

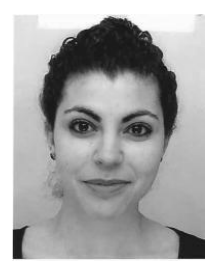

Myriam Maïza was born in Paris, France on March 24 1991. Myriam Maïza obtained a French scientist baccalauréat when she was studying at Racine in Paris, France in 2009. M. Maïza obtained a French master's degree in financial engineering from ECE Paris in 2015 after five years' study in Paris, France. While studying, Myriam Maïza had the opportunity to study in two other countries. She studied computer science at Concordia University in Montreal, Quebec in Canada in 2012 and international business at University of California Irvine in Irvine, California United States of America in 2014.

She did an internship at Raymond james International in Paris as an equity broker assistant during four months in 2014. She is currently working at BNP Paris Securities Services in Paris, France as an equity lending trader assistant since six months ago. Last year she worked on a smart beta investment project that achieve to the creation of software which gives advice to retail investors.

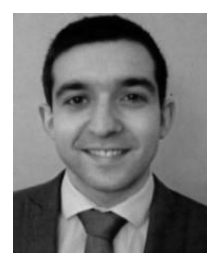

Adrien Clavel was born in Meudon, France on March 31, 1992. Adrien Clavel obtained a French scientist baccalauréat when he was studying at Anita Conti in Bruz, France in 2010. A. Clavel obtained a French master's degree in financial engineering from ECE Paris in 2015 after three years's study in Paris, France. Before these three years, Adrien Clavel was with the Scientific Prep School at Chateaubriand, Rennes. While studying, Adrien Clavel had the opportunity to study in another country. He studied international business at the Dublin Business School, Dublin, Ireland.

He did an internship at Ageas France in asset management as an assistant asset manager during four months in 2014. He is currently working at Credit Agricole Corporate \& Investment Bank, France in securitization as an assistant portfolio manager. Last year he worked on a paper, which was published in an international meeting at Singapore entitled "Dynamic shock bank liquidity analysis". 


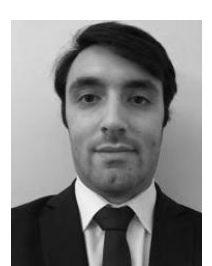

Nathan Hatem was born in Paris, France on January 29, 1991. Nathan Hatem obtained a French scientist Baccalauréat when he was studying at Notre-Dame de Sion in Paris, France in 2010; N. Hatem obtained a French master's degree in financial engineering from ECE Paris in 2015 after five years' study in Paris, France. During these five years, Nathan Hatem had the opportunity to study in two other countries. He studied computer science at Concordia University in Montreal, Quebec in Canada in 2012 and international business at University of California Irvine in Irvine, California United States of America.

He did an internship at Makor Securities as an equity sales assistant during four months in 2014. He is currently working at BFT Gestion a subsidiary of Amundi asset management in Paris, France as a diversified funds manager assistant since six months. Last year he worked on a smart beta investment project that achieves to the creation of software, which gives advice to retail investors.

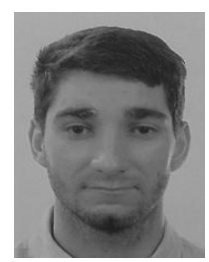

Cédric Recommande was born in Creil, France on December 27, 1991. Cédric Recommande obtained a French scientist Baccalauréat when he was studying at Auguste Renoir in Asnières sur Seine, France in 2010. C. Recommandé obtained a French master's degree in financial engineering from ECE Paris in 2015 after three years' study in Paris, France. Before these three years, Cédric Recommandé was in scientific prep school at Carnot, Paris. While studying, Cedric Recommandé had the opportunity to study in one other country. He studied international business at University of California Irvine in Irvine, California United States of America.

He did an internship at BNP PARIBAS Personal Finance on legal department as a technical support during four months in 2014. He is currently working at Banque de France in Paris, France on control management department as a modeler financial indicator since six months. Last year he worked on a paper, which was published in an international meeting at Singapore entitled "Dynamic shock bank liquidity analysis". 